\title{
Unraveling the Correlation between Solvent Properties and Sulfur Redox Behavior in Lithium-Sulfur Batteries
}

\author{
Qi He, ${ }^{1, z}$ Yelena Gorlin, ${ }^{1, a, *}$ Manu U. M. Patel, ${ }^{1}$ Hubert A. Gasteiger, ${ }^{1, * *}$ \\ and Yi-Chun $\mathrm{Lu} \mathbb{1}^{2, *, z}$ \\ ${ }^{I}$ Chair of Technical Electrochemistry, Department of Chemistry and Catalysis Research Center, Technical \\ University of Munich, Munich, Germany \\ ${ }^{2}$ Electrochemical Energy and Interfaces Laboratory, Department of Mechanical and Automation Engineering, \\ The Chinese University of Hong Kong, Hong Kong
}

\begin{abstract}
Systematic understanding of how solvent property influences Li-S redox chemistry is required to develop an effective electrolyte for $\mathrm{Li}-\mathrm{S}$ batteries. In this study, we investigate the correlation between solvent property and $\mathrm{Li}-\mathrm{S}$ redox chemistry in nine non-aqueous electrolyte solvents that cover a wide range of three main solvent physiochemical properties, namely dielectric constant $(\varepsilon)$, Gutmann donor number (DN), and acceptor number (AN). We exploit various analytical techniques including cyclic voltammetry, rotating ring disk electrode technique, UV-Vis spectroscopy and galvanostatic measurement in a two-compartment cell. We show that the potential of $\mathrm{S}_{8}$-reduction increases with increasing $\mathrm{AN}$ and that the polysulfide-reduction/oxidation is strongly influenced by the DN. The common discrepancy in the literature on the role of dielectric constant and donor number is addressed by examining the redox reactions, polysulfide stability, and the effect of salt concentration in acetonitrile - a solvent with high dielectric constant and low DN. We show that the DN is the primary descriptor for polysulfide redox reactions, as it controls the effective charge density of the solvated cation $\left(\mathrm{Li}^{+}\right)$, which affects the stability of polysulfides with different charge density via Pearson's Hard Soft Acid Base theory.

(C) The Author(s) 2018. Published by ECS. This is an open access article distributed under the terms of the Creative Commons Attribution 4.0 License (CC BY, http://creativecommons.org/licenses/by/4.0/), which permits unrestricted reuse of the work in any medium, provided the original work is properly cited. [DOI: 10.1149/2.0991816jes]

(cc) BY
\end{abstract}

Manuscript submitted October 8, 2018; revised manuscript received December 10, 2018. Published December 29, 2018.

In the search for high energy density and inexpensive post Li-ion batteries, lithium-sulfur ( $\mathrm{Li}-\mathrm{S})$ batteries have been intensively researched due to their high theoretical capacity $\left(\approx 1675 \mathrm{mAh} / \mathrm{g}_{\text {sulfur }}\right)$, high natural abundance, and non-toxicity of elemental sulfur. ${ }^{-6}$ The reversible conversion (described in Reaction 1) between elemental sulfur $\left(\mathrm{S}_{8}\right)$ and lithium sulfide $\left(\mathrm{Li}_{2} \mathrm{~S}\right)$ in a Li-S battery is normally associated with a series of intermediates, namely polysulfides $\left(\mathrm{Li}_{2} \mathrm{~S}_{\mathrm{n}}\right.$, $2 \leq \mathrm{n} \leq 8$ ), which are believed to be soluble in common organic solvents and in the state-of-the-art glyme-based electrolyte mixture, i.e. 1,2-dimethoxyethane (DME):1,3-dioxolane (DOL) $(1: 1, \mathrm{v}: \mathrm{v}){ }^{1}$

$$
\begin{aligned}
S_{8} & +16 e^{-}+16 L i^{+} \leftrightarrow \frac{8}{n} L i_{2} S_{n}+a L i^{+}+a e^{-} \leftrightarrow \frac{8}{m} L i_{2} S_{m} \\
+b L i^{+}+b e^{-} & \leftrightarrow 8 L i_{2} S
\end{aligned}
$$

where $a=\frac{16(n-1)}{n}, b=\frac{16(m-1)}{m}$, and $2 \leq m<n \leq 8$.

The cycling performance of Li-S batteries suffers from capacity fading and low efficiency, owing to the dissolution of active materials (elemental sulfur, polysulfides) into the electrolyte and diffusion away from the cathode host material, followed by their continuous depletion at the anode. ${ }^{1,2}$ In addition, the practical gravimetric energy density of Li-S batteries is limited due to the generally used high electrolyte/sulfur ratio. Therefore, developing an effective electrolyte to control the active material dissolution and to reduce the electrolyte/sulfur ratio has been one of the most critical objectives to improve the performance of Li-S batteries. ${ }^{2-4,7-9}$

The search and development for effective electrolytes require fundamental understanding of how solvent properties affect the Li-S redox chemistry. ${ }^{5,10,11}$ The selection of solvent strongly influences the stability of polysulfides which consequently affects the chemical equilibrium between various polysulfides, e.g. chain-breaking reactions (e.g. $\mathrm{S}_{6}{ }^{2-} \leftrightarrow 2 \mathrm{~S}_{3}{ }^{\bullet-}$ ) ${ }^{12}$ and/or disproportionation reactions (e.g. $\left.\mathrm{S}_{8}{ }^{2-} \leftrightarrow \mathrm{S}_{6}{ }^{2-}+\frac{1}{4} \mathrm{~S}_{8}\right) .5,10-15$ However, the primary solvent property that dictates the stability of polysulfides and its role in Li-S battery performance are still under debate. Here, the stability refers to polysulfide's

\footnotetext{
*Electrochemical Society Member.

**Electrochemical Society Fellow.

${ }^{\text {a }}$ Present Address: Research and Technology Center, Robert Bosch LLC, California, USA.

zE-mail: qi.he@tum.de;yichunlu@mae.cuhk.edu.hk
}

stability against disproportionation instead of side-reactions with solvents. The dielectric constant $(\varepsilon)$ of the solvent was suggested to be the primary descriptor governing the stability of polysulfides, ${ }^{5,8}$ as evidenced by the clear distinction of polysulfide phases in high-dielectric solvents (blue colored solutions, indicative of the presence of $\mathrm{S}_{3}{ }^{\bullet-}$ radical, ${ }^{12}$ exemplified by dimethyl sulfoxide (DMSO), dimethylacetamide (DMA), dimethylformamide (DMF), or acetonitrile (ACN)) and in low-dielectric solvents (yellow colored solutions, indicative of $\mathrm{S}_{4}{ }^{2-}$, exemplified by tetrahydrofuran (THF), DOL, and DME). Similarly, Bieker et al. ${ }^{16}$ employed UV-Vis spectroscopy to examine the chemically prepared " $\mathrm{Li}_{2} \mathrm{~S}_{8}$ " and " $\mathrm{MgS} \mathrm{S}_{8}$ " polysulfides in different solvents (note that the stoichiometry in these cases only represents an overall average value), and concluded that the dielectric constant would be the primary descriptor determining polysulfide stability. They reported that high-dielectric solvents such as DMSO, DMF and ACN are observed to stabilize long-chain polysulfides $\left(\mathrm{S}_{8}{ }^{2-}\right.$ and/or $\left.\mathrm{S}_{6}{ }^{2-}\right)$ and the $\mathrm{S}_{3}{ }^{--}$radical, while short-chain polysulfides $\left(\mathrm{S}_{4}{ }^{2-}\right)$ dominate in low-dielectric solvents such as THF, DME, and tetraethylene glycol dimethyl ether (TEGDME).$^{16}$ On the other hand, Cuisinier et al. ${ }^{17}$ showed that high EPD (electron pair donor) solvents exhibit significantly different behaviors from low EPD solvents and proposed that high EPD solvents (e.g. DMA) may be beneficial for redox-flow type $\mathrm{Li}$-S batteries, as they can prevent electrode from passivating by $\mathrm{Li}_{2} \mathrm{~S}$ formation during cycling due to the facile phase transition from $\mathrm{Li}_{2} \mathrm{~S}$ to polysulfides. In addition, Zou et al. ${ }^{10}$ studied the cyclic voltammetry of elemental sulfur in two different solvents using operando UV-Vis spectroscopy, revealing that the dominant sulfur reaction intermediate in the high-DN solvent DMSO is the $\mathrm{S}_{3}{ }^{\bullet-}$ radical, while that in the low-DN solvent mixture DOL:DME is the $\mathrm{S}_{4}{ }^{2-}$ polysulfide.

These studies are inconclusive in resolving the primary descriptor for polysulfide stability due to the issues (1) that high (low) dielectric solvents in most cases have high (low) donor number, and (2) that the type of salt and its concentration are typically neglected in these discussions. In the present work, we systematically study the Li-S redox chemistry in nine non-aqueous solvents, covering a wide range of three major solvent properties, viz., in dielectric constant $(\varepsilon)$, in Gutmann donor number (DN), and in acceptor number (AN) (s. Table 1 ), representing the polarity/polarizability (e.g. Z, $\pi^{*}$ ), Lewis basicity $(\beta)$, and Lewis acidity (e.g. $\mathrm{E}_{T}{ }^{N}$ ) of the solvent, respectively. ${ }^{18} \mathrm{We}$ exploit a well-defined three-electrode setup to conduct cyclic voltammetry $(\mathrm{CV})$ and rotating ring disk electrode (RRDE) measurements 

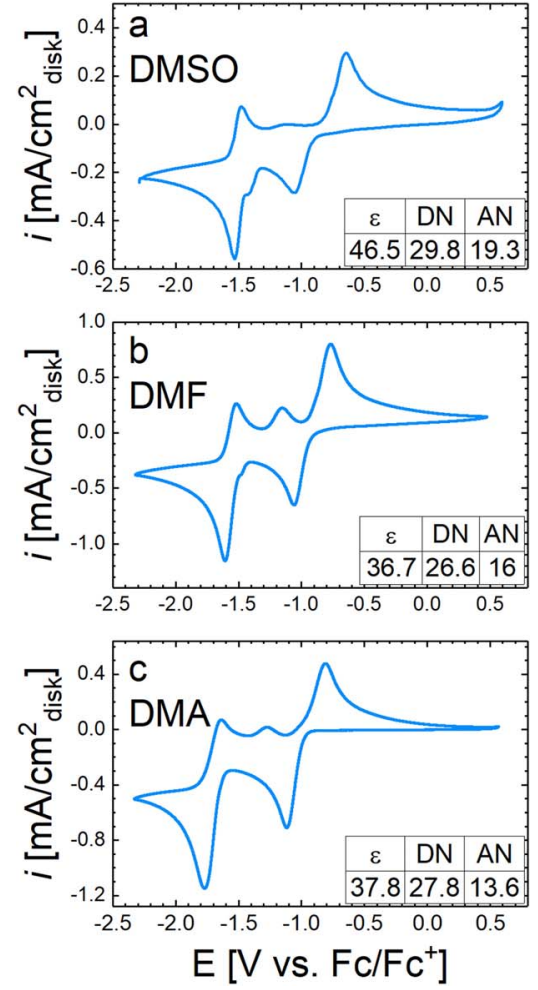
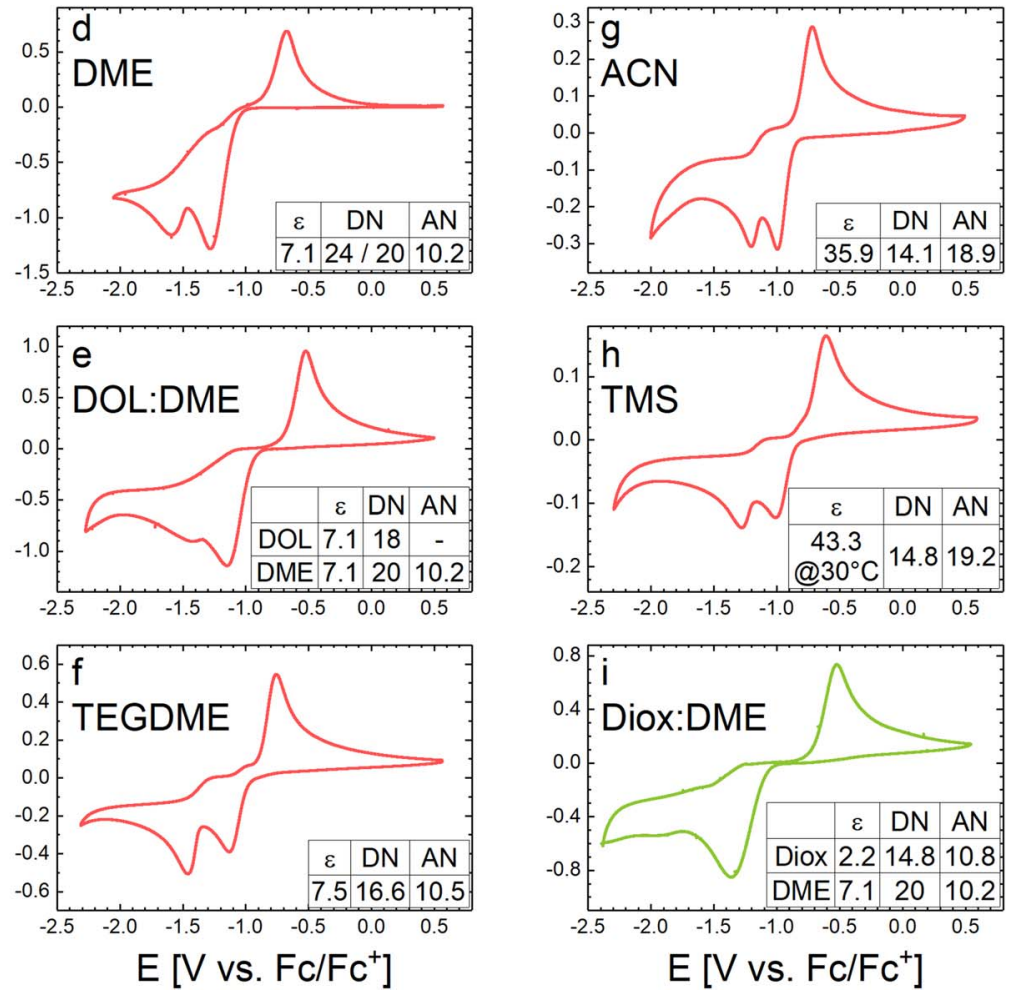

Figure 1. CVs recorded at $50 \mathrm{mV} / \mathrm{s}$ on a glassy carbon $(\mathrm{GC})$ electrode under argon atmosphere at room temperature $\left(25 \pm 1^{\circ} \mathrm{C}\right)$ in various organic solvents with dissolved $\mathrm{S}_{8}$ : (a) $2 \mathrm{mM} \mathrm{S}_{8}$ in $1 \mathrm{M}$ LiTFSI in DMSO; (b) $3 \mathrm{mM} \mathrm{S}$ in $1 \mathrm{M} \mathrm{LiTFSI} \mathrm{in} \mathrm{DMF;} \mathrm{(c)} \approx 4 \mathrm{mM} \mathrm{S}$ in 1 M LiTFSI in DMA; (d) $4 \mathrm{mM} \mathrm{S}$ in $1 \mathrm{M} \mathrm{LiTFSI} \mathrm{in}$ DME; (e) $4 \mathrm{mM} \mathrm{S}_{8}$ in $1 \mathrm{M}$ LiTFSI in DOL:DME (1:1, v:v); (f) $4 \mathrm{mM} \mathrm{S}_{8}$ in $1 \mathrm{M}$ LiTFSI in TEGDME; (g) $0.5 \mathrm{mM} \mathrm{S} \mathrm{S}_{8}$ in $1 \mathrm{M} \mathrm{LiTFSI}$ in ACN; (h) $2 \mathrm{mM} \mathrm{S} \mathrm{S}_{8}$ in $1 \mathrm{M}$ LiTFSI in TMS; and (i) 4 mM S 8 in 1 M LiTFSI in Diox:DME (1:1, v:v).

in order to study how each solvent property affects the Li-S redox reactions; Furthermore, we employ UV-Vis spectroscopy to examine the primary solvent property that dictates the stability of polysulfides. To better deconvolute the effects of $\varepsilon$ and DN which are either both high or both low for most solvents, we further study the effect of the type and concentration of salt on polysulfide stability in ACN, a solvent exhibiting a high $\varepsilon$ (35.9) which is similar to DMSO or DMF but demonstrating a low DN (14.1) which is similar to glymes. Thereby, we could decouple the influence of DN from $\varepsilon$ and $\mathrm{AN}$ on polysulfide stability, which resolved the discrepancy in the literature in determining the primary descriptor for polysulfide stability. Detailed correlations between each solvent property and the Li-S redox behavior as well as the underlying mechanisms affecting the performance of Li-S batteries will be discussed.

\section{Results and Discussion}

Correlation between $\mathrm{Li}-\mathrm{S}$ redox behavior (CV features) and solvent properties. - Figure 1 shows the cyclic voltammograms (CVs) of dissolved $\mathrm{S}_{8}$ in nine representative solvents (or solvent mixtures), including DMSO, DMA, DMF, DOL, DME, TEGDME, ACN, sulfolane (TMS) and 1,4-dioxane (Diox). We converted the recorded potential to the $\mathrm{Fc} / \mathrm{Fc}^{+}$scale in order to eliminate solvent's influence on the $\mathrm{Li} / \mathrm{Li}^{+}$redox potential. ${ }^{5}$ As is quite apparent from Fig. 1, three groups of distinct $\mathrm{CV}$ patterns were observed which can be categorized based on (i) the number of oxidation and reduction peak, and (ii) the peak separation of the reduction peaks. Group 1: CVs recorded in DMSO, DMF, and DMA largely resemble each other and are consistent with the CVs reported in literature (DMSO $\left.,{ }^{15,19} \mathrm{DMF},{ }^{20} \mathrm{DMA}\right),{ }^{19}$ having three oxidation peaks and two reduction peaks with large peak separation $(\Delta \mathrm{E}>500 \mathrm{mV}$, see Figs. 1a-1c, CVs in blue). Group 2: CVs recorded in DME, DOL:DME (1:1,v:v), TEGDME, ACN, and TMS, showing only one oxidation peak and two reduction peaks with small peak separation $(\Delta \mathrm{E} \approx 200-300 \mathrm{mV}$, see Figs. $1 \mathrm{~d}-1 \mathrm{~h}, \mathrm{CVs}$ in red).
The $2^{\text {nd }}$ reduction peak in DOL:DME is less clear at $50 \mathrm{mV} / \mathrm{s}$ but is quite pronounced at a slow scan rate of $5 \mathrm{mV} / \mathrm{s}$ (Fig. 2c). Group 3: The CV recorded in Diox:DME (1:1, v:v) shows only one oxidation peak and only one reduction peak (see Fig. 1i, CV in green), which is consistent with $\mathrm{CV}$ collected at a slow scan rate of $5 \mathrm{mV} / \mathrm{s}$ as indicated in Fig. S1.

Several studies have reported similar results for DOL:DME, ${ }^{5,10}$ $\mathrm{DME}^{19}$ and $\mathrm{ACN},{ }^{19}$ while to the best of our knowledge CVs on a planar carbon electrode in TEGDME, TMS, and Diox:DME are not available in the literature. Qualitatively, we attribute the Group 1 to high-DN solvents, and Groups 2 and 3 to low-DN solvents. Although the solvents in Group 1 exhibit high values of DN, $\varepsilon$, and $\mathrm{AN}$, we propose that actually DN would be the primary descriptor instead of $\varepsilon$ or $\mathrm{AN}$, because $\mathrm{ACN}$ which has a high $\varepsilon$ and a high AN belongs to Group 2 and thus no trend can be established using $\varepsilon$ or AN. We note that, Diox:DME (1:1, v:v) in Group 3 not only has a low DN but also the lowest dielectric constant $\varepsilon(\approx 4$, roughly estimated based on the work of Hall et al. $)^{21}$ among all solvents. It is known that a solvent (mixture) with a dielectric constant approaching one would lead to an incompletely salt (charges) dissociation. ${ }^{22}$ Therefore, in group 3 , the anion (polysulfide) is believed to strongly associate with cation and consequently, it would have less interaction with solvent molecules. That is, solvent molecules would have less impact on polysulfide stability via dipole-ion interaction (AN, DN) compared to it in a highly dissociated system. In addition to these CV classifications, we will further examine the correlations between each solvent property $(\varepsilon, \mathrm{AN}, \mathrm{DN})$ and (i) the onset potential of $\mathrm{S}_{8}$-reduction, and (ii) the reversibility of the $2^{\text {nd }}$ oxidation peak.

The onset potential of $S_{8}$-reduction vs. AN of the solvent.-A positive correlation was found between the onset potential of $S_{8}$-reduction and the AN of solvents (Fig. 2a), i.e. the higher the AN of the solvent, the higher the onset potential of $\mathrm{S}_{8}$-reduction. The onset potentials of $\mathrm{S}_{8}$ reduction were taken from the $1^{\text {st }}$ derivative of the current density 

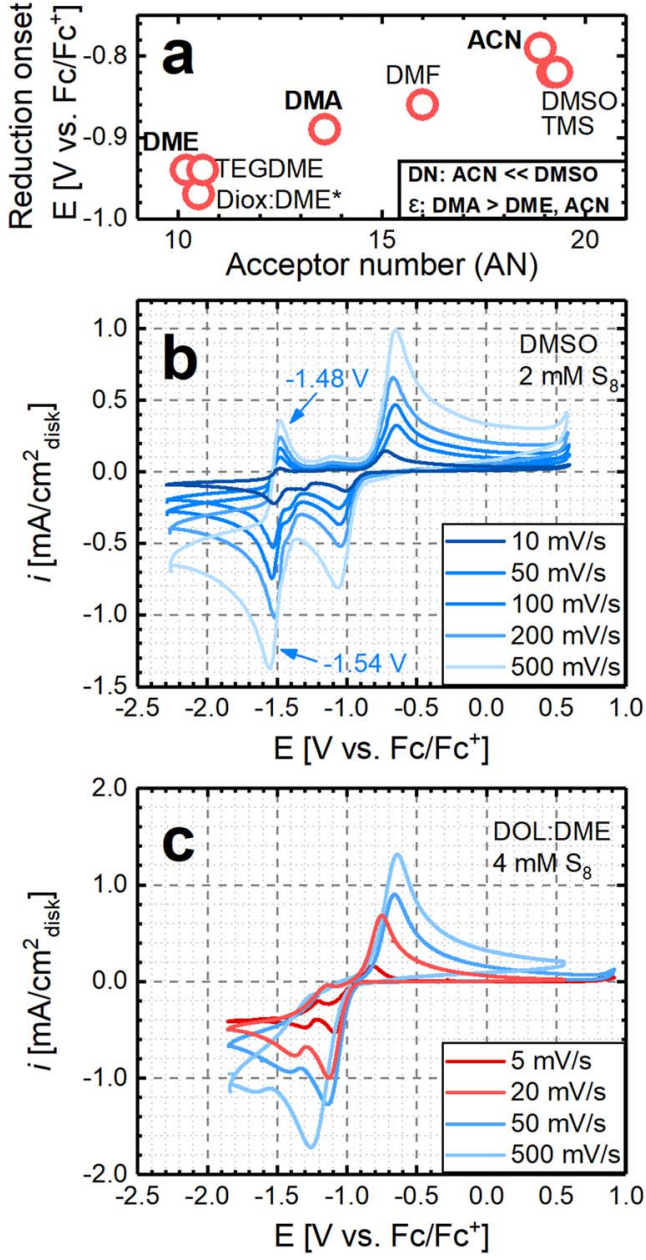

Figure 2. a) Correlation between acceptor number and the onset potential for the reduction of $S_{8}$ in different solvents (obtained from the CVs in Fig. 1 using a procedure outlined in Fig. S2), with the inset comparing the DN and the dielectric constant for selected solvents. Only eight solvents are shown in Fig. 2a, as no acceptor number for DOL is available in literature; b) iRcorrected CVs of a GC electrode in $1 \mathrm{M}$ LiTFSI in DMSO with $2 \mathrm{mM} \mathrm{S}_{8}$ at various scan rates (the peak potentials at $500 \mathrm{mV} / \mathrm{s}$ of the $2^{\text {nd }}$ redox pair are marked); c) iR-corrected CVs of a GC electrode in $1 \mathrm{M} \mathrm{LiTFSI} \mathrm{in} \mathrm{DOL:DME}$ $(1: 1, \mathrm{v}: \mathrm{v})$ with $4 \mathrm{mM} \mathrm{S}_{8}$ at various scan rates.

(Fig. S2). The acceptor number was introduced by Mayer et al.,23 seeking to empirically describe the electrophilic property of a solvent. The acceptor number is generally derived from ${ }^{31} \mathrm{P}-\mathrm{NMR}$ measurements of triethylphosphine oxide dissolved in the respective solvents and is considered as an indicator for the ability of the solvent to solvate anions. The first step of $S_{8}$-reduction is generally agreed to be the reduction of elemental $S_{8}$ to $S_{8}{ }^{2-.5,10,15,24,25}$

$$
S_{8}+2 e^{-} \rightarrow S_{8}^{2-}
$$

The solvation energy of the $\mathrm{S}_{8}{ }^{2-}$ anion in high-AN solvents is greater than that in low-AN solvents due to the stronger electrophilic property of the high-AN solvents, which leads to a more positive reduction potential of $\mathrm{S}_{8}$ to $\mathrm{S}_{8}{ }^{2-}$ in high-AN solvents than in low-AN solvents (see. Equation 3).

$$
\begin{aligned}
E & \sim f\left(E^{\circ}+\frac{R T}{n F} \ln K\right) \sim f\left(\Delta G^{\circ}\right) \sim f \\
& \left(\text { solvation energy of anion }\left(\text { e.g. } S_{8}{ }^{2-}\right)\right) \sim f(A N)
\end{aligned}
$$

Our observation is consistent with Mayer et al., ${ }^{23}$ showing that the $\mathrm{AN}$ of the solvent influences the half-wave potential (polargarphic) of the reduction of $\left[\mathrm{Bu}_{4} \mathrm{~N}\right]_{3}\left[\mathrm{Fe}(\mathrm{CN})_{6}\right]$ (tetrabutylammonium hexacyanoferrate), which can also be rationalized using Equation 3.

The reversibility of the $2^{\text {nd }}$ oxidation peak $v s . D N$ of the solvent.- - It is clear from Fig. 1 that the second redox pair $\left(\mathrm{at} \approx-1.5 \mathrm{~V}_{\mathrm{Fc}}\right)$ is more reversible in solvents with high DN (e.g. DMSO, DMA, DMF, see Figs. 1a-1c, blue CVs) and appears irreversible in solvents with low DN (e.g. DME, DOL:DME, TEGDME, TMS, ACN, and Diox:DME). Note that there is no ambiguity between DN and dielectric constant, as this redox pair is irreversible in ACN (low DN, high $\varepsilon$ ). Due to the limited sample size and the inaccuracy of DN describing the solvation ability of solvent to $\mathrm{Li}^{+}$, it is not our intention to draw a linear correlation between $\mathrm{DN}$ and $\mathrm{CV}$ features. One is only able to qualitatively couple $\mathrm{CV}$ features such as oxidation peak number and reduction peak separation with the range of DN of the solvent, rather than with other properties such as AN and dielectric constant. Taking DMSO (high DN) as an example, the second redox couple exhibits a one-electron reversible process, as evidenced from the scan rate independent peak separation of $\approx 60 \mathrm{mV}$ (Fig. 2b). ${ }^{26}$ The assignment of the $2^{\text {nd }}$ redox pair in DMSO has been extensively discussed in the literature. After the $1^{\text {st }}$ reduction peak, during which $S_{8}$ is electrochemically reduced, other polysulfides are generated by disproportionation reactions as described in Equations 4, 5 and 6. ${ }^{15,25,27,28}$

$$
\begin{gathered}
S_{8}+2 e^{-} \rightarrow S_{8}^{2-} \\
S_{8}^{2-} \leftrightarrow S_{6}^{2-}+\frac{1}{4} S_{8} \\
S_{6}^{2-} \leftrightarrow 2 S_{3}^{\bullet-}
\end{gathered}
$$

The $2^{\text {nd }}$ redox pair has been assigned to the electrochemical reduction and oxidation reaction between $\mathrm{S}_{3}{ }^{\bullet-} / \mathrm{S}_{3}{ }^{2-},{ }^{28-30}$ as shown in Equation 7.

$$
S_{3}^{\bullet-}+e^{-} \rightarrow S_{3}^{2-}
$$

Zou et al. ${ }^{10}$ have applied operando UV-Vis spectroscopy to show that the UV-Vis absorbance of $\mathrm{S}_{3}{ }^{--}(620 \mathrm{~nm})$ decreases significantly as the electrode potential goes below $\sim-1.5 \mathrm{~V}_{\mathrm{Fc}}$ and increases drastically as soon as the electrode potential reverses, suggesting that the $\mathrm{S}_{3}{ }^{\bullet-} / \mathrm{S}_{3}{ }^{2-}$ redox process is relatively reversible. This is consistent with our observation of a fast and reversible $2^{\text {nd }}$ redox peak observed in DMSO (Fig. 2b).

In contrast, the oxidation reaction of the $2^{\text {nd }}$ redox pair is much less reversible in low-DN solvents, resulting in a less visible oxidation peak (Figs. 1d-1h). In addition, the potential of the $2^{\text {nd }}$ reduction peak shifts strongly with scan rate in a low-DN solvent like DOL:DME (Fig. 2c), indicating the irreversibility of the $2^{\text {nd }}$ redox process. Interestingly, the related oxidation peak can be clearly observed at the slow scan rate of $5 \mathrm{mV} / \mathrm{s}$ (located at $-1.15 \mathrm{~V}_{\mathrm{Fc}}$, see Fig. 2c), indicating that the oxidation process exhibits sluggish electrode kinetics rather than poor chemical stability. The $2^{\text {nd }}$ redox pair in DOL:DME has been assigned to the reduction of $\mathrm{S}_{4}{ }^{2-}$ based on the decreasing UV-Vis absorption at $420 \mathrm{~nm}\left(\mathrm{~S}_{4}{ }^{2-}\right) \cdot{ }^{10}$ However, no increase of absorbance at other wavelength was observed in the operando UV-Vis study. ${ }^{10}$ This suggests that $\mathrm{S}_{4}{ }^{2-}$ is electrochemically reduced to form species that are UV-Vis inactive (e.g. solid), which can be generalized to ${ }^{10,11,14,31}$

$$
S_{4}^{2-}+n e^{-}+m L i^{+} \leftrightarrow L_{2} i_{2} / L i_{2} S
$$

In addition, considering the sluggish kinetics observed in low-DN solvents, we believe that this redox pair involves the formation of solid phases such as $\mathrm{Li}_{2} \mathrm{~S}_{2} / \mathrm{Li}_{2} \mathrm{~S}$ from $\mathrm{S}_{4}{ }^{2-}$. Consistent behavior was also observed in other solvents (Fig. S3), showing that the $2^{\text {nd }}$ oxidation peak is much more visible/reversible in high-DN solvents, e.g. DMF $(\mathrm{DN}=26.6)$ and DMA ( $\mathrm{DN}=27.8)$, compared to low-DN solvents, e.g. $\mathrm{ACN}(\mathrm{DN}=14.1)$

In addition to the $\mathrm{CV}$ analyses, we also exploited the rotating ring disk electrode technique to investigate the kinetics and $\mathrm{Li}-\mathrm{S}$ reaction 


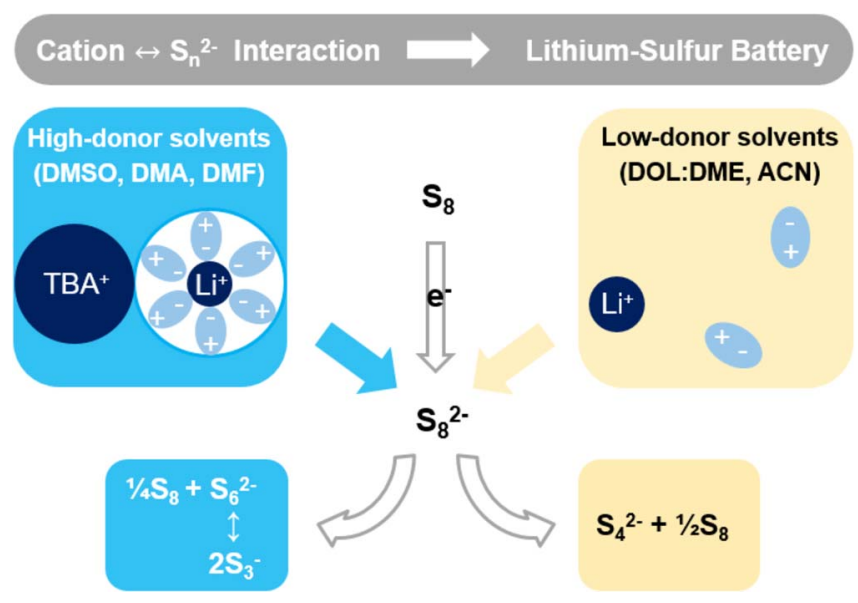

Scheme 1. Summary of how polysulfide stability/speciation is affected by its interaction with cations and solvent molecules.

mechanism in various electrolytes, as we had done previously for DMSO and DOL:DME-based electrolytes. ${ }^{5}$ We observed that electrolytes with high-DN solvents and electrolytes with $\mathrm{TBA}^{+}$cation (a strongly solvating cation) have always two well-defined reduction plateaus, among which the $1^{\text {st }}$ reduction wave was always accompanied with an electron transfer of $\sim 1.7 \mathrm{e}^{-} / \mathrm{S}_{8}$, estimated by applying the Levich-equation (see Fig. S4). This suggests a two-electron reduction of $S_{8}$ to $S_{8}{ }^{2-}$ (Eq. 3), indicating $S_{8}{ }^{2-}$ is more stabilized in such electrolyte environments, that is, with high-DN solvent and/or with strongly solvating cation $\left(\mathrm{TBA}^{+}\right) ., 32$

Other CV analyses such as peak current evaluation using the Randle-Sevcik equation are further illustrated in the supporting information (Fig. S5 and S6). The parameters used in the Levich equation for the analysis of the RRDE data, which are also useful for numerical modeling of Li-S batteries (e.g. the diffusion coefficient of dissolved $\mathrm{S}_{8}$ in various electrolytes) are presented in the supporting information (Table S2). In short, two clear correlations between solvent properties and $\mathrm{CV}$ features were identified: 1) the onset potential of the first $\mathrm{S}_{8}$-reduction peak increases with increasing $\mathrm{AN}$, and 2) the reversibility of the second oxidation peak increases with increasing DN, owing to the formation of soluble polysulfides, rather than solid phase polysulfides, in high-DN solvents.

Why the donor number is the primary descriptor for polysulfide redox chemistry?-Here we employ UV-Vis spectroscopy to examine the polysulfide stability in different solvents and we show that the stability of various polysulfides $\left(\mathrm{S}_{\mathrm{n}}{ }^{2-}\right)$ is mainly dictated by the solvated cations, which can be manipulated by solvent donor number (DN), cation concentration, and the cation type $\left(\mathrm{Li}^{+}, \mathrm{TBA}^{+}\right)$, as illustrated in Scheme 1.

Effect of solvent donor number--Fig. 3a shows the UV-Vis spectra of a $5 \mathrm{mM}$ concentration of polysulfides with a nominal stoichiometry of " $\mathrm{Li}_{2} \mathrm{~S}_{8}$ " in nine different solvents with $1 \mathrm{M}$ LiTFSI. Clearly, high-DN solvents (blue lines labeled g-i) all have a strong absorption at 620 $\mathrm{nm}$ (corresponding to $\left.\mathrm{S}_{3}{ }^{\bullet-}\right), 475 \mathrm{~nm}\left(\mathrm{~S}_{6}{ }^{2-}\right), 492 \mathrm{~nm}\left(\mathrm{~S}_{8}{ }^{2-}\right)$, and 350 $\mathrm{nm}\left(\mathrm{S}_{6}{ }^{2-}\right),{ }^{10,13,15,16,25}$ while low-DN solvents (red lines labeled a-f) all have similarly pronounced absorption maxima at $420 \mathrm{~nm}\left(\mathrm{~S}_{4}{ }^{2-}\right)$ without any absorption at $620 \mathrm{~nm}\left(\mathrm{~S}_{3}{ }^{-}\right)$. We note that TEGDME with lower DN of 16.6 has a higher absorption at $620 \mathrm{~nm}$ compared to $\mathrm{DME}(\mathrm{DN} \approx 20$ or 24 ), which can be explained by 1 ) the chelate effect, the known cage structure formed by TEGDME that can better solvate $\mathrm{Li}^{+}$cation ${ }^{33,34}$ and 2) that donor number, an empirical parameter derived from the solvation of $\mathrm{SbCl}_{5}$, cannot fully represent the solvation of $\mathrm{Li}^{+} .{ }^{35}$ The distinct speciation of polysulfides in different solvents are consistent with the literature and can be well explained by the Pearson's Hard Soft Acid Base (HSAB) theory. ${ }^{36}$ The Li ions solvated in high-DN solvents (strongly solvated $\mathrm{Li}^{+}$, soft acid) preferentially stabilize polysulfides with lower charge density
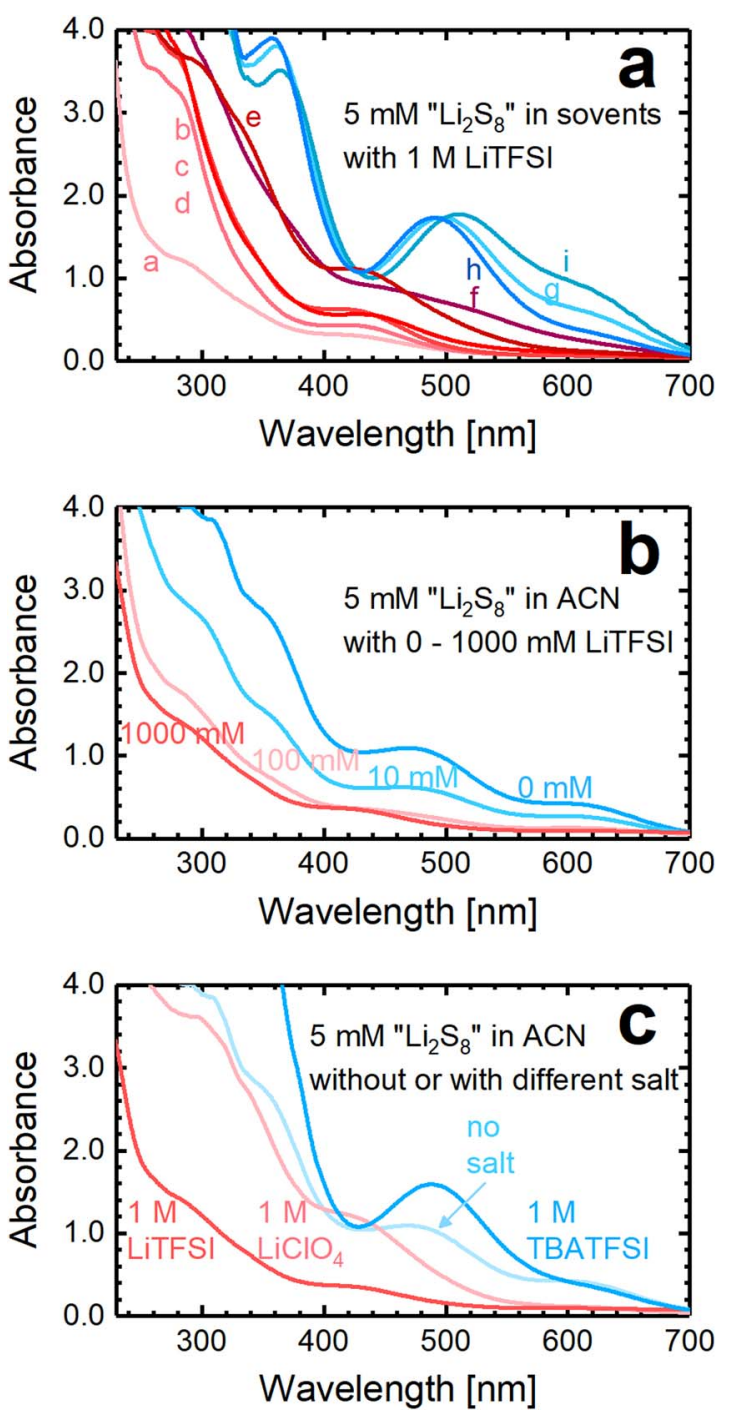

Figure 3. Ex-situ UV-Vis spectra of various electrolytes with a $5 \mathrm{mM}$ concentration of polysulfides with a nominal stoichiometry of " $\mathrm{Li}_{2} \mathrm{~S}_{8}$ " with different conductive salts and salt concentrations. a) UV-Vis spectra in the presence of $1 \mathrm{M}$ LiTFSI in nine different solvents: six low-DN solvents, namely (a) ACN (incompletely dissolved " $\mathrm{Li}_{2} \mathrm{~S}_{8}$ "), (b) DME, (c) DOL:DME (1:1, v:v), (d) Diox:DME (1:1, v:v), (e) TMS, (f) TEGDME, as well as three high-DN solvents, namely (h) DMSO, (i) DMA, and (g) DMF. b) UV-Vis spectra in ACN with different concentration of LiTFSI or without LiTFSI: $0 \mathrm{mM}$ (blue), $10 \mathrm{mM}$ (light blue), $100 \mathrm{mM}$ (light red), and $1000 \mathrm{mM}$ (red). c) UV-Vis spectra in ACN without added salt or with different salts: $1 \mathrm{M}$ TBATFSI (blue), no added salt (light blue), $1 \mathrm{M} \mathrm{LiClO}_{4}$ (light red), and $1 \mathrm{M} \mathrm{LiTFSI} \mathrm{(red).}$

(softer base, e.g. $\mathrm{S}_{8}{ }^{2-}, \mathrm{S}_{6}{ }^{2-}, \mathrm{S}_{3}{ }^{--}$), whereas the Li ions solvated in low-DN solvents (poorly solvated $\mathrm{Li}^{+}$, hard acid) preferentially stabilize polysulfides with higher charge density (harder base, e.g. $\mathrm{S}_{4}{ }^{2-}$ ). Here, we compare the hardness/softness (i.e. charge density) of different polysulfides based on reported theoretical studies. Both Steudel et al. ${ }^{37}$ and Pascal et al. ${ }^{38}$ have calculated the charge density of terminal and in-chain sulfur atoms in different polysulfides (summarized in Fig. S7). Although Steudel et al. ${ }^{37}$ have focused their work on sodium-sulfur system at elevated temperature $(600 \mathrm{~K})$, their calculations were also conducted at $298 \mathrm{~K}$ to address the structure and atomic charges of isolated polysulfide dianions and anion radicals at $298 \mathrm{~K}$. It was reported that regardless of the assumed nature of the continuum medium (e.g. vacuum or polarizable continuum model with dielectric constants 8 and 78), the negative charge density of terminal sulfur atoms in $\mathrm{S}_{\mathrm{x}}{ }^{2-}$-chains decreases in the following order 
$\mathrm{S}_{2}{ }^{2-}>\mathrm{S}_{3}{ }^{2-}>\mathrm{S}_{4}{ }^{2-}>\mathrm{S}_{5}{ }^{2-}>\mathrm{S}_{6}{ }^{2-}>\mathrm{S}_{7}{ }^{2-}>\mathrm{S}_{8}{ }^{2-}$; in addition, it was calculated that that the negative charge density of $\mathrm{S}_{3}{ }^{\bullet-}$ would be even lower than that reported for $\mathrm{S}_{8}{ }^{2-}$ (see comment to Fig. S7 in the SI), ${ }^{37,38}$ Similar trend was also reported by Pascal et al., ${ }^{38}$ who calculated the average valence electron populations for the internal and terminal sulfur atom of $\mathrm{Li}_{2} \mathrm{~S}_{\mathrm{x}}$ in a 12 TEGDME molecule environment (see Fig. S7). Although the absolute value of the negative charge density is different in different studies and also depends on the assumed surrounding continuum (see Fig. S7), the overall trend is still the same in all studies, namely that short-chain polysulfides (e.g. $\mathrm{S}_{4}{ }^{2-}$ ) have a higher negative charge density on the terminal sulfur atoms than that in longchain polysulfides (e.g. $\mathrm{S}_{8}{ }^{2-}$ ) and in the $\mathrm{S}_{3}{ }^{\bullet-}$ radical. In other words, the negative charge is more strongly delocalized on long-chain polysulfides $\left(\mathrm{S}_{8}{ }^{2-}\right)$ and on $\mathrm{S}_{3}{ }^{\bullet-}$ radicals compared to that on short-chain polysulfides $\left(\mathrm{S}_{4}{ }^{2-}\right)$, which in turn implies that the softness of polysulfides decreases in the following order: $\mathrm{S}_{3}{ }^{\bullet-}>\mathrm{S}_{8}{ }^{2-}>\mathrm{S}_{6}{ }^{2-}>\mathrm{S}_{4}{ }^{2-}>$ $\mathrm{S}_{3}{ }^{2-}>\mathrm{S}_{2}{ }^{2-}$. Therefore, the $\mathrm{S}_{4}{ }^{2-}$ polysulfide (harder base) is better stabilized by weakly solvated $\mathrm{Li}^{+}$(hard acid) prevalent in low-DN solvents, whereas $\mathrm{S}_{3}{ }^{\bullet-}, \mathrm{S}_{8}{ }^{2-}$, and $\mathrm{S}_{6}{ }^{2-}$ (softer base) are better stabilized by strongly solvated $\mathrm{Li}^{+}$(soft acid) prevalent in high-DN solvents.

To confirm that the polysulfide stability is mainly affected by donor number rather than by the dielectric constant $(\varepsilon)^{5,16}$ or the acceptor number (AN), we further investigated the polysulfide speciation in a rather unique solvent $(\mathrm{ACN})$ which has a low donor number (DN = 14.1, similar as TEGDME) but high dielectric constant $(\varepsilon=36)$ and acceptor number (AN $=18.9)$ which are close to those of DMSO (which, however, has also a very high DN; see Table S1). In Fig. 3a, we show that the speciation of polysulfides in ACN resembles that in the low-DN system (TEGDME) despite ACN has a completely different $\varepsilon$ and AN compared to TEGDME. This clearly suggests that dielectric constant and acceptor number are not the critical parameters governing polysulfide stability/speciation in the presence of a high concentration of $\mathrm{Li}^{+}$cations.

Effect of $\mathrm{Li}^{+}$concentration.- In contrary to our results, Bieker et al. ${ }^{16}$ reported that the UV-Vis spectrum of a nominal " $\mathrm{Li}_{2} \mathrm{~S}_{8}$ " stoichiometry in ACN resembles that of " $\mathrm{Li}_{2} \mathrm{~S}_{8}$ " in DMSO, and it was thus concluded that $\varepsilon$ is the primary descriptor for polysulfide stability/speciation rather than DN. After careful analysis, we believe this discrepancy is related to a difference in the $\mathrm{Li}^{+}$concentration. In our work, $1 \mathrm{M}$ LiTFSI was added to the $5 \mathrm{mM}$ " $\mathrm{Li}_{2} \mathrm{~S}_{8}$ " dissolved in ACN to mimic the practical Li-S battery environment, whereas no additional salt was used for the preparation of the 1 and $10 \mathrm{mM}^{\text {" }} \mathrm{Li}_{2} \mathrm{~S}_{8}$ " containing ACN solution in the work by Bieker et al. ${ }^{16}$ To resolve this question, we evaluated UV-Vis spectra of $5 \mathrm{mM}$ " $\mathrm{Li}_{2} \mathrm{~S}_{8}$ " in ACN with different concentrations of LiTFSI and without added LiTFSI (Fig. 3b). The sample of " $\mathrm{Li}_{2} \mathrm{~S}_{8}$ " in ACN without added LiTFSI salt (light blue) indeed shows a similar spectrum as " $\mathrm{Li}_{2} \mathrm{~S}_{8}$ " in DMSO (line $\mathrm{h}$ in Fig. 3a), with a high absorption at 620,475 , and $350 \mathrm{~nm}$, quite analogous to what was reported by Bieker et al. ${ }^{16}$ On the other hand, the solution of " $\mathrm{Li}_{2} \mathrm{~S}_{8}$ " in ACN with $1 \mathrm{M}$ LiTFSI presents a similar absorption spectrum as " $\mathrm{Li}_{2} \mathrm{~S}_{8}$ " in low-DN TEGDME and DOL:DME, with a pronounced absorption maximum at $420 \mathrm{~nm}$. This experiment confirms our hypothesis that the $\mathrm{Li}^{+}$concentration is responsible for the apparent discrepancy between our data and those by Bieker et al. ${ }^{16}$ We believe that when no additional "naked" $\mathrm{Li}^{+}$is present, soft polysulfides (e.g. $\mathrm{S}_{3}{ }^{--}$) can be stabilized by the surrounding ACN molecules owing to the high charge accepting ability of ACN (high AN of 18.9). That is, at extremely low cation concentrations, the stability of polysulfides is governed by the solvent molecules via dipole-anion interaction (solvent $\left.-\mathrm{S}_{\mathrm{n}}{ }^{2-}\right)$, instead of the cation-anion interaction $\left(\mathrm{Li}^{+}\right.$(solvents) $-\mathrm{S}_{\mathrm{n}}{ }^{2-}$ ). However, with increasing $\mathrm{Li}^{+}$concentration, the number of "naked" $\mathrm{Li}^{+}$(hard acid) increases and the influence of the cation on polysulfide stability becomes significant, which shifts the equilibrium to stabilize hard polysulfides (e.g. $\mathrm{S}_{4}{ }^{2-}$ ). As shown in Fig. 3b, with increasing $\mathrm{Li}^{+}$concentration $(0 \mathrm{M} \rightarrow 1 \mathrm{M})$, the absorption at $620 \mathrm{~nm}$ $\left(\mathrm{S}_{3}{ }^{--}\right)$and at $475 \mathrm{~nm}\left(\mathrm{~S}_{6}{ }^{2-}\right) / 492 \mathrm{~nm}\left(\mathrm{~S}_{8}{ }^{2-}\right)$ decreases gradually, while the absorption at $420 \mathrm{~nm}\left(\mathrm{~S}_{4}{ }^{2-}\right)$ increases. This experiment confirms our hypothesis and resolves the apparent discrepancy in the literature with regards to the speciation of polysulfides in ACN solvent.
Recently, several research groups have proposed a sparingly solubilizing electrolyte system for Li-S batteries, ${ }^{4,39,40}$ aiming to limit the concentration of polysulfides on the order of $1 \mathrm{mM}$ or less by occupying most of the solvent molecules with supporting salt, leaving none left to coordinate to polysulfide molecules. ${ }^{4}$ In addition, a high concentration of Li-salts are required to achieve sparingly solubilizing electrolyte such as $\mathrm{ACN}_{2}$ LiTFSI-TTE $^{39}$ and $\mathrm{ACN}_{2}$-LiTFSI-HFE. ${ }^{40}$ We have learned from Fig. $3 b$ that more "naked" $\mathrm{Li}^{+}$in the system will shift the polysulfides equilibrium to the one with higher charge density, namely shorter chain polysulfides (e.g. $\mathrm{S}_{4}{ }^{2-}$ ). Although the solvation state of polysulfides are likely different in sparingly solubilizing electrolytes, the impact of $\mathrm{Li}^{+}$cation on the polysulfide stability/speciation may still apply, that shorter chain polysulfides are preferably promoted by the cation-anion interaction in a highly concentrated electrolyte.

Effect of cation type.-To further show that cations can very strongly affect polysulfide speciation, we replaced the $1 \mathrm{M} \mathrm{Li}^{+}$cations (a hard acid) with $1 \mathrm{M} \mathrm{TBA}^{+}$cations (a soft acid regardless of solvent) ${ }^{41}$ in the solution of " $\mathrm{Li}_{2} \mathrm{~S}_{8}$ " in ACN. As shown in Fig. 3c, the spectrum of " $\mathrm{Li}_{2} \mathrm{~S}_{8}$ " in ACN-TBATFSI resembles that in DMSO with $1 \mathrm{M} \mathrm{LiTFSI}$ (line $\mathrm{h}$ in Fig. 3a) where long-chain polysulfides are clearly dominating (e.g. $\left.620 \mathrm{~nm}\left(\mathrm{~S}_{3}{ }^{\bullet-}\right), 475 \mathrm{~nm}\left(\mathrm{~S}_{6}{ }^{2-}\right), 492 \mathrm{~nm}\left(\mathrm{~S}_{8}{ }^{2-}\right)\right)$. This further confirms that large concentrations of cations and their type will affect the speciation of polysulfides.

Li-S cell charge/discharge behavior in two-compartment cells.To further distinguish the impact of donor number, dielectric constant, and acceptor number on the charge/discharge behavior of Li-S cells, we employ a two-compartment cell with a lithium metal anode and a cathode either based on a dissolved $\mathrm{S}_{8}$ containing catholyte or based on a solid $\mathrm{S}_{8} / \mathrm{C}$ composite cathode. The two-compartment cell is a closed system that confines polysulfides in the cathode and allows full reactions (long reaction time in hours), whereas $\mathrm{CV}$ and RRDE were conducted in an open system where polysulfides are freely to diffuse in a large amount of electrolyte (short reaction time in seconds). With this, we evaluate the Li-S cell behavior in $\mathrm{ACN}$ ( $\mathrm{DN}=14.1, \varepsilon=$ $35.95, \mathrm{AN}=18.9)$ and compare it first to that in DMSO ( $\mathrm{DN}=29.8$, $\varepsilon=46.5, \mathrm{AN}=19.3)$ and then to that in commonly used electrolyte DOL $(\mathrm{DN}=18, \varepsilon=7.13)$ : $\mathrm{DME}(\mathrm{DN}=24, \varepsilon=7.1, \mathrm{AN}=10.2)$.

Figs. $4 \mathrm{a}$ and $4 \mathrm{~b}$ show the voltage profiles of the catholyte (0.5 $\mathrm{mM} \mathrm{S}_{8}$ ) two-compartment cell in ACN (green) and DMSO (blue) at $0.5 \mathrm{C}$ and $2.9 \mathrm{C}$ (based on $1672 \mathrm{mAh} / \mathrm{g}_{\mathrm{s}} \equiv 1 \mathrm{C}$ ). Despite the fact that $\mathrm{ACN}$ and DMSO exhibit very similar dielectric constant and acceptor number, the catholyte two-compartment cell voltage profiles and discharge/charge potentials in ACN are significantly different from that in DMSO. The voltage plateau separation in the two-compartment cell is found to be well-correlated to the separation of the reduction peaks in cyclic voltammograms (Fig. 1). The significantly different voltage profiles observed in DMSO and ACN cannot be explained by their almost identical dielectric constant and acceptor number, but can be correlated to their differences in DN (ACN (14.1) vs DMSO (29.8)) as already discussed above. Consistently, DMSO-alike voltage profiles were also reported for other high-DN solvents, such as DMA (DN = $27.8)^{11}$ and DMF $(\mathrm{DN}=26.6) .{ }^{10}$

In contrast, the cell voltage profiles with $\mathrm{ACN}$ based electrolyte is very similar to that in the low-DN solvent DOL:DME (average $\mathrm{DN} \sim 20$ ). Even using conventional $\mathrm{S}_{8} / \mathrm{C}$ composite cathodes (albeit with relatively low loadings of $0.25-0.6 \mathrm{mg}_{\mathrm{s}} / \mathrm{cm}^{2}$ ), the voltage profiles obtained in ACN and DOL:DME based electrolytes are still very similar (Fig. 4c; note that the larger hysteresis for high-loaded sulfur electrodes is due to the higher current densities in this case, as the C-rate is kept constant). Therefore, we conclude that the donor number - governing the effective charge density of the solvated cation $\left(\mathrm{Li}^{+}\right)-$is the primary descriptor determining the voltage profile (reaction potentials and peak separations) of Li-S batteries via dictating the speciation of polysulfides in different electrolytes. Similar findings were also reported by Schneider et al., ${ }^{42}$ who observed distinctly different cell voltages in different solvents and attributed this to the considerably stronger solvation effect for small and hard lithium ions 


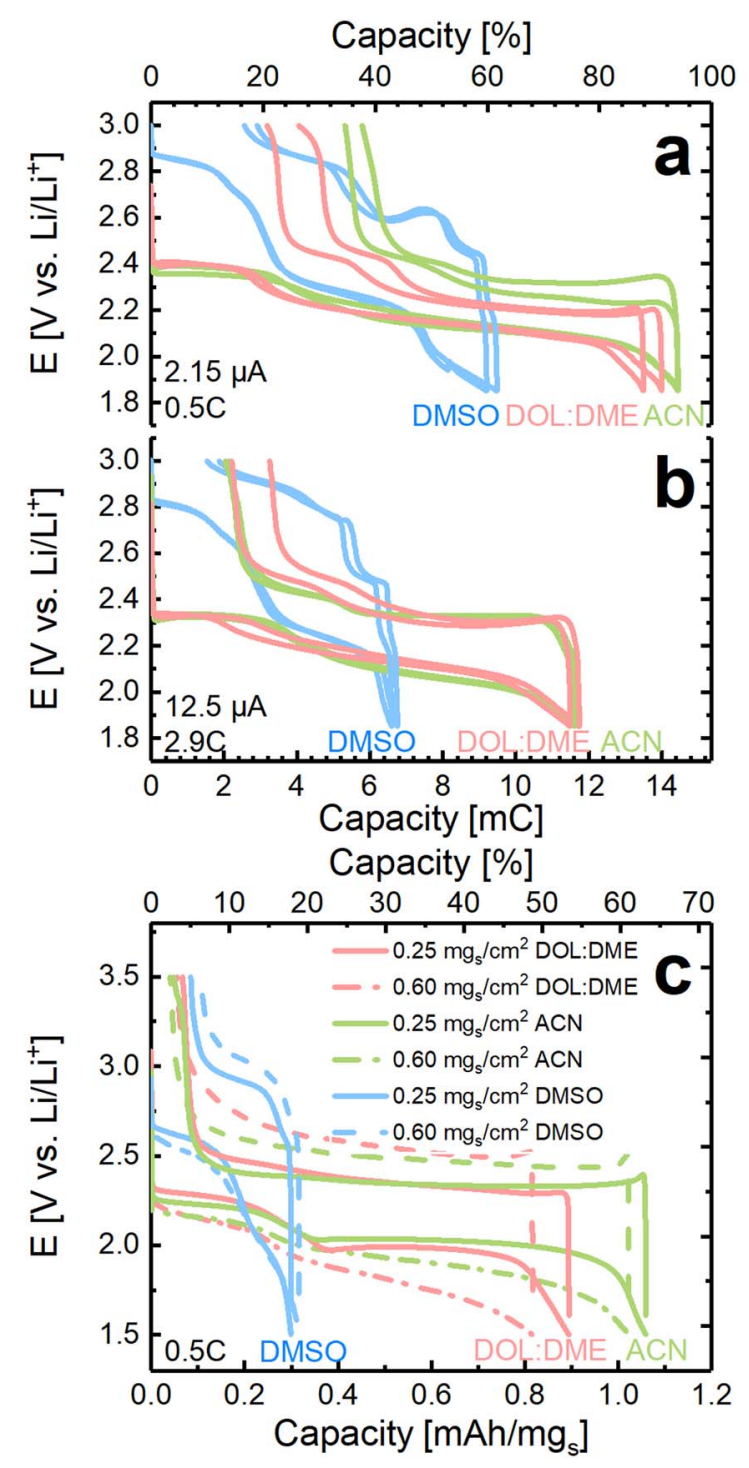

Figure 4. Galvanostatic discharge/charge profiles of sulfur cathodes either based on dissolved sulfur or based on an $\mathrm{S}_{8} / \mathrm{C}$ composite, recorded in a twocompartment cell where anode (metallic lithium) and cathode are separated by a lithium ion conducting glass membrane. a) Charge/discharge at $2.15 \mu \mathrm{A}$ $(\equiv 0.5 \mathrm{C})$ and $\mathrm{b})$ at $12.5 \mu \mathrm{A}(\equiv 2.9 \mathrm{C})$ of a $\mathrm{Li}-\mathrm{S}$ catholyte cell with a cathode based on a carbon fiber paper filled with $20 \mu \mathrm{L}$ of $0.5 \mathrm{mM} \mathrm{S}_{8}$ (corresponding to a theoretical capacity of $15.4 \mathrm{mC}$ ) and $0.6 \mathrm{M}$ LiTFSI with various solvents: DOL:DME (1:1, v:v) in red, ACN in green, and DMSO in blue (anolyte in all cases: $0.6 \mathrm{M}$ LiTFSI in DOL:DME $(1: 1, \mathrm{v}: \mathrm{v}))$. c) Galvanostatic discharge/charge profiles of $\mathrm{S}_{8} / \mathrm{C}$ composites (sulfur loadings are given in the figure) at $0.5 \mathrm{C}$ with $1.5 \mathrm{M}$ LiTFSI containing catholytes: DOL:DME (1:1, v:v) in red, ACN in green, and DMSO in blue (anolyte in all cases: $1.5 \mathrm{M}$ LiTFSI in DOL:DME $(1: 1, \mathrm{v}: \mathrm{v}))$.

(indicated by the DN) compared to that for the polysulfide anions (indicated by AN).

Lastly, the discharge capacity achieved in $\mathrm{ACN}$ is similar to that of DOL:DME, which is higher than it is in DMSO. However, the discharge capacity of Li-S cells in DMSO is also much lower compared to that in other high-DN solvents such as $\mathrm{DMF}^{10}$ and DMA ${ }^{11}$ measured under comparable conditions. Therefore, we believe that the limited discharge capacity in DMSO is related to its high viscosity $(1.99 \mathrm{cp})^{18}$ compared to that of other solvents $\operatorname{DMF}(0.79 \mathrm{cp}),{ }^{18} \mathrm{DMA}(0.93 \mathrm{cp}),{ }^{18}$ $\operatorname{ACN}(0.34 \mathrm{cp}),{ }^{18}$ and DOL:DME (average $\left.\sim 0.5 \mathrm{cp}\right),{ }^{18}$ which significantly reduces the mobility of $\mathrm{Li}^{+}$cations and soluble polysulfides and thus limits the growth of solid lithium sulfide in the electrode. Based on our observations, solvent viscosity show limited effect on polysulfide stability/speciation and the electrochemical redox behavior. Despite TMS $(\sim 10.07 \mathrm{cp}){ }^{18}$ has a much higher viscosity than ACN $(\sim 0.34 \mathrm{cp}),{ }^{18}$ they both have shown similarity in redox behavior i.e. $\mathrm{CV}$ features (Figs. 1g, 1h, oxidation/reduction peak numbers, reduction peak separation, reduction onset potential and the reversibility of $2^{\text {nd }}$ oxidation peak) and polysulfide stability/speciation represented by UV-Vis spectra (Fig. 3a). We believe that viscosity mainly affects the transport behavior of polysulfide, which has great importance to the cell performance, such as rate capability (shown in Fig. 4) and ohmic resistance affecting energy efficiency. On the other hand, the polysulfide stability/speciation is determined by the cation-anion interaction and the dipole-anion interaction. In the nine solvents investigated, we observed that the cation-anion interaction has a stronger impact on the polysulfide stability over dipole-anion interaction. Therefore, we propose donicity would be the primary descriptor (but not the only one) determining the redox behavior of polysulfides in a well dissociated electrolyte system (reasonable dielectric constant required), and acceptor number can also regulate the redox pair, as exemplified by its influence on reduction onset potential (Fig. 2a).

In addition to solvent and the concentration of salt, the type of anions has been shown to influence the sulfur redox pair, as anions with different donicity may also contribute to the coordination of $\mathrm{Li}^{+} .43-45$ For instance, Watanabe and co-workers ${ }^{43}$ have shown a strong anionic effects on polysulfide solubility in solvate ionic liquid electrolytes, which is directly related to polysulfide shuttling. Furthermore, a strong influence of anions on the reaction intermediates were reported in the Li-O $\mathrm{O}_{2}$ system where $\mathrm{NO}_{3}{ }^{-}$with higher donicity is believed to assist the solvation of $\mathrm{Li}^{+}$, resulting in an increased capacity and toroid formation. ${ }^{46}$ Efforts in understanding anion effect in Li-S redox chemistry and cell behavior are on-going and will be reported in future work.

\section{Conclusions}

In summary, we study the correlation between solvent property and Li-S redox chemistry in nine nonaqueous electrolyte solvents that cover a wide range of dielectric constant $(\varepsilon)$, donor number (DN) and acceptor number (AN). We reveal that the potential of the $S_{8} / S_{8}{ }^{2-}$ redox process increases with increasing $\mathrm{AN}$ of the solvent, which could be attributed to a higher solvation energy of $\mathrm{S}_{8}{ }^{2-}$ in high-AN solvents. We show that the donicity of the solvent (expressed in donor number, DN) is the primary solvent property controlling polysulfide redox reactions, as it dictates the effective charge density of the solvated cation $\left(\mathrm{Li}^{+}\right)$, which affects the stability and thus the speciation of the various polysulfides via Pearson's Hard Soft Acid Base theory. The commonly encountered discrepancy in the literature on the role of dielectric constant and donor number is addressed by examining the redox reactions and the polysulfide speciation as function of salt concentration and type in acetonitrile, a solvent with high dielectric constant and low donor number. Our study shows that the interaction between the cation-complex with the polysulfide anions is the most critical factor controlling the sulfur/polysulfide redox reactions and the polysulfide speciation (Scheme 1), which can be tailored via the donicity of the solvent (DN) as well as via the concentration and the type of cations in the sulfur-based rechargeable batteries.

\section{Acknowledgments}

The German Federal Ministry for Economic Affairs and Energy is acknowledged for funding under the auspices of the "LiMo" project (funding number 03ET6045D). Y. Gorlin gratefully acknowledges the support of the Alexander von Humboldt Postdoctoral fellowship and Carl Friedrich von Siemens Fellowship Supplement.

\section{ORCID}

Yelena Gorlin (ID https://orcid.org/0000-0002-9242-8914

Yi-Chun Lu (D) https://orcid.org/0000-0003-1607-1615 


\section{References}

1. M. Barghamadi, A. S. Best, A. I. Bhatt, A. F. Hollenkamp, M. Musameh, R. J. Rees, and T. Rüther, Energy Environ. Sci., 7, 3902 (2014).

2. S. Zhang, K. Ueno, K. Dokko, and M. Watanabe, Adv. Energy Mater., 5, 1500117 (2015).

3. D. Eroglu, K. R. Zavadil, and K. G. Gallagher, J. Electrochem. Soc., 162, 982 (2015).

4. L. Cheng, L. A. Curtiss, K. R. Zavadil, A. A. Gewirth, Y. Shao, and K. G. Gallagher, ACS Energy Lett., 1, 503 (2016).

5. Y.-C. Lu, Q. He, and H. A. Gasteiger, J. Phys. Chem. C, 118, 5733 (2014).

6. D. Zheng, G. Wang, D. Liu, J. Si, T. Ding, D. Qu, X. Yang, and D. Qu, Adv. Mater Technol., 3 (2018).

7. Y. Chen, H. Zhang, W. Xu, X. Yang, Y. Yu, X. Li, and H. Zhang, Adv. Funct. Mater., 28, 1 (2018).

8. G. Zhang, H.-J. Peng, C.-Z. Zhao, X. Chen, L.-D. Zhao, P. Li, J.-Q. Huang, and Q. Zhang, Angew. Chemie Int. Ed., 1 (2018).

9. Z. Li, Y. Zhou, Y. Wang, and Y. C. Lu, Adv. Energy Mater., 1802207 (2018).

10. Q. Zou and Y.-C. Lu, J. Phys. Chem. Lett., 7, 1518 (2016).

11. Y. Gorlin, A. Siebel, M. Piana, T. Huthwelker, H. Jha, G. Monsch, F. Kraus, H. A. Gasteiger, and M. Tromp, J. Electrochem. Soc., 162, A1146 (2015).

12. T. Chivers and P. J. W. Elder, Chem. Soc. Rev., 42, 5996 (2013).

13. C. Barchasz, F. Molton, C. Duboc, J. C. Leprêtre, S. Patoux, and F. Alloin, Anal. Chem., 84, 3973 (2012).

14. M. Cuisinier, P.-E. Cabelguen, S. Evers, G. He, M. Kolbeck, A. Garsuch, T. Bolin, M. Balasubramanian, and L. F. Nazar, J. Phys. Chem. Lett., 4, 3227 (2013).

15. R. P. Martin, W. H. Doub, J. L. Roberts, and D. T. Sawyer, Inorg. Chem., 12, 1921 (1973).

16. G. Bieker, J. Wellmann, M. Kolek, K. Jalkanen, M. Winter, and P. M. Bieker, Phys. Chem. Chem. Phys., 19, 11152 (2017).

17. M. Cuisinier, C. Hart, M. Balasubramanian, A. Garsuch, and L. F. Nazar, Adv. Energy Mater, 5, 1401801 (2015)

18. D. Aurbach, Nonaqueous Electrochemistry, Marcel Dekker, Inc., New York, NY, (1999).

19. Y. Jung, S. Kim, B.-S. Kim, D.-H. Han, S.-M. Park, and J. Kwak, Int. J. Electrochem. Sci., 3, 566 (2008).

20. F. Gaillard, E. Levillain, and J. P. Lelieur, J. Electroanal. Chem., 432, 129 (1997)

21. D. S. Hall, J. Self, and J. R. Dahn, J. Phys. Chem. C, 119, 22322 (2015)

22. A. Abbott, Chem. Soc. Rev., 22, 435 (1993).

23. U. Mayer, V. Gutmann, and W. Gerger, Monatshefte fuer Chemie, 106, 1235 (1975).
24. P. Leghi, J. Lelieur, and E. Levillain, Electrochem commun., 4, 406 (2002).

25. R. Bonnaterre and G. Cauquis, J. Chem. Soc. Chem. Commun., 0, 293 (1972).

26. A. J. Bard and L. R. Faulkner, Electrochemical Methods Fundamentals and Applications, 2nd ed., John Wiley \& Sons, INC, New York, NY, (2000).

27. T. Fujinaga, T. Kuwamoto, S. Okazaki, and M. Hojo, Bull. Chem. Soc. Jpn., 53, 2851 (1980).

28. M. Cuisinier, C. Hart, M. Balasubramanian, A. Garsuch, and L. F. Nazar, Adv. Energy Mater, 5, 1401801 (2015).

29. B.-S. Kim and S.-M. Park, J. Electrochem. Soc., 140, 115 (1993).

30. J. Paris and V. Plichon, Electrochim. Acta, 26, 1823 (1981).

31. S. Waluś, C. Barchasz, R. Bouchet, J.-C. Leprêtre, J.-F. Colin, J.-F. Martin, E. Elkaïm, C. Baehtz, and F. Alloin, Adv. Energy Mater, 5, 1500165 (2015).

32. Q. Zou, Z. Liang, G. Du, C. Liu, E. Y. Li, Y. Lu, Q. Zou, Z. Liang, G. Du, C. Liu, E. Y. Li, and Y. Lu, J. Am. Chem. Soc., 140, 10740 (2018).

33. C. Zhang, A. Yamazaki, J. Murai, J. Park, T. Mandai, K. Ueno, K. Dokko, and M. Watanabe, J. Phys. Chem. C, 118, 17362 (2014).

34. I. Gunasekara, S. Mukerjee, E. J. Plichta, M. A. Hendrickson, and K. M. Abraham, J. Electrochem. Soc., 162, A1055 (2015).

35. V. Gutmann, Electrochim. Acta, 21, 661 (1976)

36. T.-L. Ho, Chem. Rev., 75, 1 (1975).

37. R. Steudel and Y. Steudel, Chem. -A Eur. J., 19, 3162 (2013).

38. T. A. Pascal, K. H. Wujcik, J. Velasco-Velez, C. Wu, A. A. Teran, M. Kapilashrami, J. Cabana, J. Guo, M. Salmeron, N. Balsara, and D. Prendergast, J. Phys. Chem. Lett., 5, 1547 (2014).

39. C. Lee, Q. Pang, S. Ha, L. Cheng, S. Han, K. R. Zavadil, K. G. Gallagher, L. F. Nazar, and M. Balasubramanian, ACS Cent. Sci., 3, 605 (2017).

40. M. Cuisinier, P.-E. Cabelguen, B. D. Adams, A. Garsuch, M. Balasubramanian, and L. F. Nazar, Energy Environ. Sci., 7, 2697 (2014)

41. C. O. Laoire, S. Mukerjee, K. M. Abraham, E. J. Plichta, and M. A. Hendrickson, J. Phys. Chem. C, 114, 9178 (2010).

42. H. Schneider, C. Gollub, T. Weiss, J. Kulisch, K. Leitner, R. Schmidt, M. M. Safont-Sempere, Y. Mikhaylik, T. Kelley, C. Scordilis-Kelley, M. Laramie, and H. Du, J. Electrochem. Soc., 161, A1399 (2014).

43. K. Ueno, J.-W. Park, A. Yamazaki, T. Mandai, N. Tachikawa, K. Dokko, and M. Watanabe, J. Phys. Chem. C, 117, 20509 (2013).

44. H. Chen and Y. C. Lu, Adv. Energy Mater., 6, 1 (2016).

45. H. J. Peng, J. Q. Huang, X. Y. Liu, X. B. Cheng, W. T. Xu, C. Z. Zhao, F. Wei, and Q. Zhang, J. Am. Chem. Soc., 139, 8458 (2017).

46. C. M. Burke, V. Pande, A. Khetan, V. Viswanathan, and B. D. McCloskey, Proc. Natl. Acad. Sci., 112, 9293 (2015). 